\title{
Superoxide anion production by monocytes of corticosteroid-treated asthmatic patients
}

\author{
M. Majori, I. Vachier, P. Godard, M. Farce, J. Bousquet, P. Chanez
}

Superoxide anion production by monocytes of corticosteroid-treated asthmatic patients. $M$ Majori, I. Vachier, P. Godard, M. Farce, J. Bousquet, P. Chanez. OERS Journals Ltd 1998. ABSTRACT: Reactive oxygen species play an important role in promoting inflammation. Blood monocytes have been described to release higher amounts of superoxide anion in uncontrolled and untreated asthmatics. Corticosteroids are widely used in asthma, but little is known about their molecular mechanism of action. The aim of our study was to analyse the ex vivo effect of corticosteroid treatments on superoxide anion release by blood monocytes.

Superoxide anion release was measured by a spectrophotometric method based on the superoxide dismutase (SOD) -inhibitable reduction of ferricytochrome $\mathrm{C}$ by blood monocytes from untreated patients and asthmatics treated with i.v., inhaled and oral corticosteroids.

Monocytes from uncontrolled and untreated asthmatics, released high amounts of superoxide anions. After short-term treatment with $i . v$., corticosteroids, this release was found to decrease significantly $(1.410$ versus $0.340 \mathrm{nM}, \mathrm{p}<0.05)$. Cells from asthmatics who had undergone long-term treatment with inhaled or oral steroids presented low amounts of superoxide anion production, with a significant difference as compared to untreated asthmatics $(0.375 \mathrm{nM} p<0.01$ and $0.620 \mathrm{nM} p<0.02$ respectively).

In general, patients with controlled asthma (treated with short-term oral steroids, or with long-term inhaled steroids) released lesser amounts of superoxide anion than uncontrolled and untreated asthmatics. In the case of steroid-dependent asthmatics there was no difference between patients with a controlled or uncontrolled disease. Eur Respir J 1998; 11: 133-138.

Bronchial asthma is a chronic inflammatory disease of the airways characterized by an increased recruitment of inflammatory cells, including mononuclear phagocytic cells such as monocytes [1]. The ability of these cells to generate highly reactive oxygen species (ROS), including super-oxide anion, following activation of a membraneassociated reduced nicotinamide adenine dinucleotide phosphate (NADPH) oxidase is an important part of the host defence system, and has also been implicated in inflammation in a variety of pulmonary diseases [2, 3], including asthma $[4,5]$. Oxygen metabolites cause tissue injury, smooth muscle contraction, increased airway vascular permeability, and may increase airway responsiveness and release of var-ious mediators [4].

In previous studies, it has been shown that alveolar macrophages [6], blood monocytes [5] and eosinophils [7] from asthmatic patients were able to produce high amounts of ROS. Alveolar macrophage activation was correlated with the severity of asthma [6]. This activation occurred immediately after antigen challenge, and the appearance of high-density macrophages which release higher amounts of superoxide anion occurred during the late airway response [8]. Several investigators have also suggested that polymorphonuclear leucocytes from asthmatic patients generated more ROS than control subjects $[9,10]$. This activation was correlated with the level of bronchial hyperreactivity [11].
INSERM U454, Clinique des Maladies Respiratoires, Hôpital Arnaud de Villeneuve, Montpellier, France.

Correspondence: P. Chanez

INSERM U454

Hopital Arnaud de Villeneue

Clinique des Maladies Respiratoires

34295 Montpellier Cedex 5

France

Fax: 0033467521848

Keywords: Asthma

corticosteroids

monocytes

superoxide anion

Received: December 301996

Accepted after revision September 301997

This work was partly supported by a grant from the University of Parma, Italy.
Corticosteroids are the most potent anti-inflammatory agents available for the treatment of asthma [12], but little is known about their molecular mechanism of action in improving symptoms or the way they reduce hyperresponsiveness. It has been proposed that corticosteroids inhibit the inflammatory process by redirection of lymphocyte traffic, inhibition of cytokine gene expression [13] and inhibition of the expression of adhesion molecules [14].

The effect of anti-inflammatory corticosteroids on oxygen radical production by phagocytes has been less extensively studied, and the results are contradictory. In vitro studies have demonstrated an inhibitory effect of high corticosteroid concentrations on oxygen radical production in human granulocytes [15]. In contrast, dexamethasone treatment has been reported to have no effect on ROS secretion in human blood-derived macrophages [16], but have a stimulatory effect on human monocytes [17]. It has been reported that in vivo corticosterone had no effect on murine peritoneal inflammatory cells [18]. Ex vivo inhaled steroid treatment had no effect on superoxide anion production in human alveolar macrophages $[19,20]$. In contrast, a recent study showed that in vivo administration of dexamethasone or methylprednisolone led to a dose-dependent inhibition of rat peritoneal leucocyte chemiluminescence [21]. Moreover, hydrocortisone was found to inhibit superoxide anion production during an asthma attack [22]. 
The present study was carried out to clarify the ex vivo effects of corticosteroids on superoxide anion production by blood monocytes from asthmatic patients. We compared superoxide anion production in blood monocytes from untreated asthmatic patients and three groups of asthmatic patients treated with corticosteroids. In the first group, superoxide anion release was assessed before and after short-term therapy with high-dose i.v. corticosteroids in order to test the acute effects induced by corticosteroids. The second group included patients receiving long-term therapy with inhaled corticosteroids, and the third group included patients receiving long-term therapy with inhaled and oral corticosteroids (for at least $1 \mathrm{yr}$ ) called steroid-dependent asthmatic patients.

\section{Materials and methods}

\section{Patients}

Twenty seven nonsmoking asthmatic patients, ranging $22-72$ yrs of age (mean \pm SD $48.68 \pm 15.13 \mathrm{yrs}$ ), were included in this study. Asthma was defined according to the American Thoracic Society [23] criteria, and all patients had a reversible airway disease. Informed consent was obtained from all patients in accordance with the Ethics Committee of the University of Montpellier. Patients who did not present any nocturnal asthma symptoms and who did not use any $\beta_{2}$-agonist were classified as patients with controlled disease.

Nine asthmatic patients had not undergone any treatment (group 1), and demonstrated a $12 \%$ increase in their forced expiratory volume in one second (FEV1) after inhalation of $200 \mu \mathrm{g}$ salbutamol. All these patients were symptomatic, with no anti-inflammatory treatment when entering the study. These patients were treated with a short course of $i . v$. methylprednisolone given over 10 days (80 mg.day $\left.{ }^{-1}\right)$, and demonstrated at least a $15 \%$ improvement in their morning prebronchodilator FEV1. Six patients were seen again after the treatment for evaluation but three were lost to follow-up.

Six asthmatic patients (moderate persistent asthma) included in this study had been treated with $1.5 \mathrm{mg}$ of inhaled beclomethasone dipropionate (BDP) for at least 6 months, along with $100 \mu \mathrm{g}$ salmeterol (group 2). This dose of inhaled steroid was the minimal dose required to control their symptoms.

Twelve patients with severe steroid-dependent asthma (severe persistent asthma) were included in this study (group 3). They had already been monitored in our clinic for at least $1 \mathrm{yr}$ prior to the start of the present study. They were described as steroid-dependent, since we failed to wean them from corticosteroids in the previous year and they had presented with recurrent threatening episodes of acute asthma during the past 2 yrs. They were treated on a regular basis with prednisone from $10-60 \mathrm{mg}^{- \text {day }^{-1} \text { (mean }}$ dose: $34.3 \mathrm{mg}$ ) and 1,600 $\mu \mathrm{g}$ of inhaled budesonide, 100 $\mu \mathrm{g}$ salmeterol and $10 \mathrm{mg} \cdot \mathrm{kg}^{-1}$ of long-acting theophylline, according to recently published guidelines [24].

\section{Preparation of human blood monocytes}

Blood monocytes were prepared as previously described [5]. Peripheral blood was recovered by venipuncture using heparin $\left(25 \mathrm{U} \cdot \mathrm{mL}^{-1}\right)$. Cells were isolated by centrifugation of blood samples over isotonic Percoll solutions at densities of 1.097 (Percoll 72\%) and 1.086 (Percoll $63 \%$ ). A $5 \mathrm{~mL}$ volume of a $63 \%$ Percoll solution was layered over $5 \mathrm{~mL}$ of $72 \%$ solution in a $15 \mathrm{~mL}$ conical tube. Whole blood $(5 \mathrm{~mL})$ was layered over the Percoll gradient and then centrifuged at $1,200 \times \mathrm{g}$ for $20 \mathrm{~min}$ at room temperature, resulting in the formation of one band of mononuclear cells and bands of polymorphonuclear leucocytes (PMN) above the $72 \%$ layer.

The mononuclear cell suspension in Percoll was removed and washed once with an equal volume of RPMI 1640 medium. Contaminating erythrocytes were lysed by incubation for $10 \mathrm{~min}$ in a lysing buffer solution of $130 \mathrm{mM} \mathrm{NH} \mathrm{NH}_{4} \mathrm{Cl}, 10 \mathrm{mM}$ Tris buffer, and $16 \mathrm{mM} \mathrm{K}_{2} \mathrm{CO}_{3}$, $\mathrm{pH} 7.4$, and recovered by centrifugation. Cells were then washed in RPMI 1640 medium and counted in the initial suspension. A total of $10^{5}$ blood monocytes were then incubated in 96-multiwell dishes with $100 \mu \mathrm{L}$ RPMI 1640, containing antibiotics (L-glutamine, streptomycin and penicillin) and $20 \%$ foetal calf serum (FCS), for $2 \mathrm{~h}$ at $37^{\circ} \mathrm{C}$ in a humid atmosphere of $95 \%$ air and $5 \% \mathrm{CO}_{2}$ to separate adherent monocytes from suspended lymphocytes. At the end of this incubation period nonadherent cells were then removed.

\section{Superoxide anion generation assay}

The superoxide anion concentration produced by adherent blood monocytes was quantified using a spectrophotometric method based on the superoxide dismutase (SOD)-inhibitable reduction of ferricytochrome $\mathrm{C}$, adapted for the 96-well microtitre assay, according to JoNHSTON et al. [5]. Ferricytochrome C, SOD, and phorbol 12-myristate 13-acetate (PMA) were purchased from Sigma Chemical Company (St Louis, MO, USA). Each well contained $10^{5}$ cells, and $80 \mu \mathrm{mol}$ of ferricytochrome $\mathrm{C}$ in phosphate-buffered saline (PBS) (with $\mathrm{Ca}^{2+} / \mathrm{Mg}^{2+}$ ). Superoxide anion production was measured under two conditions, spontaneously and after PMA stimulation for 90 min. PMA was freshly prepared by diluting a stock solution in PBS to a final concentration of $100 \mathrm{ng} \cdot \mathrm{mL}^{-1}$. Paired wells were established with and without SOD $\left(30 \mu \mathrm{g} \cdot \mathrm{mL}^{-1}\right.$ final concentration) to assess the specificity of cytochrome $\mathrm{C}$ reduction as control. Optical density was then determined with absorbance at $550 \mathrm{~nm}$ for $90 \mathrm{~min}$, with the results expressed in nmol. Each reaction was run in quadruplicate and measurements performed at 10, 15, 30, 45, 60 and $90 \mathrm{~min}$. The stimulatability coefficient was defined as the ratio of PMA-stimulated/nonstimulated superoxide anion production at $60 \mathrm{~min}$ after stimulation [9].

\section{Statistical analysis}

Untreated asthmatics and three groups of treated patients were compared using the analysis of variance (ANOVA), and the Mann-Whitney U-test was used for unpaired data. The Wilcoxon W-test was used to compare superoxide anion levels in patients before and after short-term methyl prednisolone treatment. The kinetic study results are ex- 
Table 1. - Characteristics of asthmatic patients and stimulatability coefficient in the superoxide anion release at 60 min

\begin{tabular}{lcccccc}
\hline Asthmatics & $\begin{array}{c}\text { Sex } \\
\text { M/F }\end{array}$ & $\begin{array}{c}\text { Age } \\
\text { yrs }\end{array}$ & $\begin{array}{c}\text { FEV1 } \\
\text { predicted }\end{array}$ & Treatment & $\begin{array}{c}\text { CS dose } \\
\text { mg.day-1 }\end{array}$ & $\begin{array}{c}\text { Stimulatability } \\
\text { coefficient mean } \\
(25-75 \% \text { percentiles })\end{array}$ \\
\hline Group 1: Untreated & $3 / 6$ & $51.3 \pm 16.4$ & $68.1 \pm 10.4$ & $\beta_{2}$ as required & - & $60(3.5-8.5)$ \\
$\quad$ after short-term & $3 / 3$ & $50.0 \pm 17.8$ & $90.8 \pm 7.8^{+}$ & i.v. CS & 80 & $3.5(1.0-4.9)$ \\
$\quad \begin{array}{l}\text { treatment } \\
\text { Group 2: Inhaled-steroids }\end{array}$ & $2 / 4$ & $56.7 \pm 14.1$ & $83.3 \pm 13.4^{\ddagger}$ & Inh CS & 1.5 & $3.0(1.0-4.7)$ \\
Group 3: Steroid-dependent & $7 / 5$ & $45.6 \pm 16.7$ & $51.4 \pm 22.1^{\dagger}$ & Inh + oral CS & $34.3 \pm 13.1$ & $6.9(1.9-17.7)$ \\
\hline
\end{tabular}

Values are expressed as mean \pm SD. M: male; F: female; FEV1: forced expiratory volume in one second. CS: corticosteroid; Inh: inhaled.

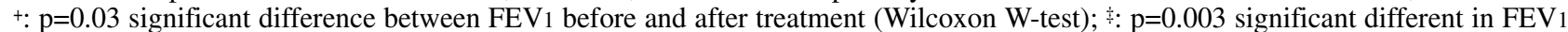
between steroid-dependent and inhaled treatment (Mann-Whitney test); $\uparrow \mathrm{p}<0.02$ significant difference in FEV 1 between untreated and inhaled treatment (Mann-Whitney U-test).

pressed as mean, and the time point analysis results expressed as median with a $25-75$ th percentile.

\section{Results}

\section{Patients}

The demographic characteristics of the groups of asthmatic patients are summarized in table 1 .

In group 1, untreated asthmatic patients (sex matched), with a mean \pm SD age of $51.3 \pm 16.4$ yrs and with an FEV1 \pm SD of $68.1 \pm 10.4 \%$ of pred value were classified as uncontrolled asthmatics requiring a course of $i$.v. corticosteroids. After 10 days of administration of $80 \mathrm{mg}$ methylprednisolone, symptoms had improved and FEV1 increased significantly in all patients, being $90.8 \pm 7.8 \%$ pred $(\mathrm{p}<0.05$ (Wilcoxon W-test)).

In group 2, patients with a mean \pm SD age of $56.7 \pm 14.1$ yrs were classified with moderate persistent asthma (sex matched), requiring long-term treatment with inhaled steroids. They were in a steady state with a mean \pm SD FEV 1 of $83.3 \pm 13.4 \%$ pred, and significantly different from untreated patients $(\mathrm{p}<0.02$ (Mann-Whitney U-test)).

Group 3 included steroid-dependent asthmatics with severe persistent asthma (sex matched), with a mean \pm SD of $45.6 \pm 16.7 \mathrm{yrs}$, requiring long-term therapy with oral steroids. Their mean \pm SD FEV1 $51.4 \pm 22.1 \%$ pred was significantly different $(p=0.003)$ from patients undergoing long-term treatment with inhaled steroids. The oral corticosteroid dose was the minimal dose required to improve their symp-toms, i.e. a mean of $34.3 \pm 13.1 \mathrm{mg}$ per day.

Forty per cent of the patients enrolled in this study were allergic, as assessed by at least three skin-prick tests positive to common allergens.

\section{Superoxide anion production analysis}

Time-kinetic studies. Time-kinetic studies were performed for up to $90 \mathrm{~min}$, and superoxide anion accumulation was measured on unstimulated and PMA-stimulated blood monocytes.

For unstimulated cells, the time-kinetic curves for superoxide anion production by blood monocytes from untreated asthmatics and the three groups of treated asthmatic patients did not show any significant differences (fig. 1). However, a nonsignificant decrease in spontaneous superoxide anion production in blood monocytes was

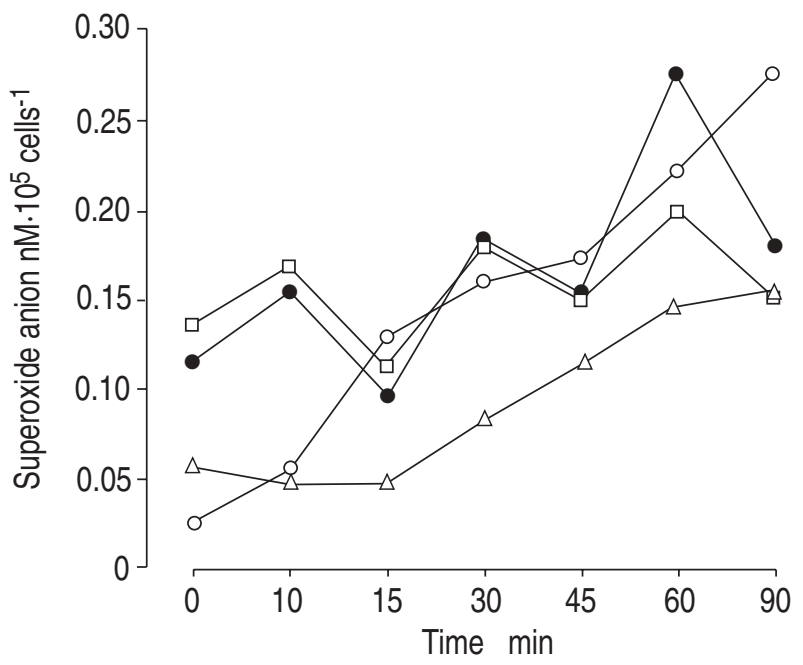

Fig. 1. - Kinetic curves for spontaneous superoxide anion production by blood monocytes. $\bullet$ : untreated; $\bigcirc$ : short-term treatment; $\square$ : steroiddependent asthmatics; $\Delta$ : patients treated with inhaled steroids. Each symbols represents the mean of four reactions performed simultaneously.

observed in asthmatic patients treated with inhaled steroids.

For PMA-stimulated cells, the time-kinetic curve for blood monocytes of untreated asthmatic patients was higher than for the three groups of treated asthmatic patients, with significant differences in superoxide anion production from 10-90 min for steroid-dependent asthmatic patients, from 15-90 min for inhaled treatment asthmatic patients, and from 30-90 min for short-term-treated asthmatic patients (fig. 2). No differences were observed in the shape of the four curves, but the slopes were very different between the treated groups (slope coefficients: 0.005 for steroid-dependent, 0.006 for inhalation treated, 0.007 for short-term-treated patients and 0.013 for the untreated group).

Time point analysis. For untreated asthmatic patients, maximum release was obtained at $60 \mathrm{~min}$. This time was chosen to study individual data for all four populations of asthmatic patients. The ratio of PMA-stimulated/spontaneous superoxide anion production from blood monocytes was calculated for each individual result at $60 \mathrm{~min}$ and represents the stimulatability coefficient (table 1).

No significant difference was observed for spontaneous production, despite a lower release for all three popula- 


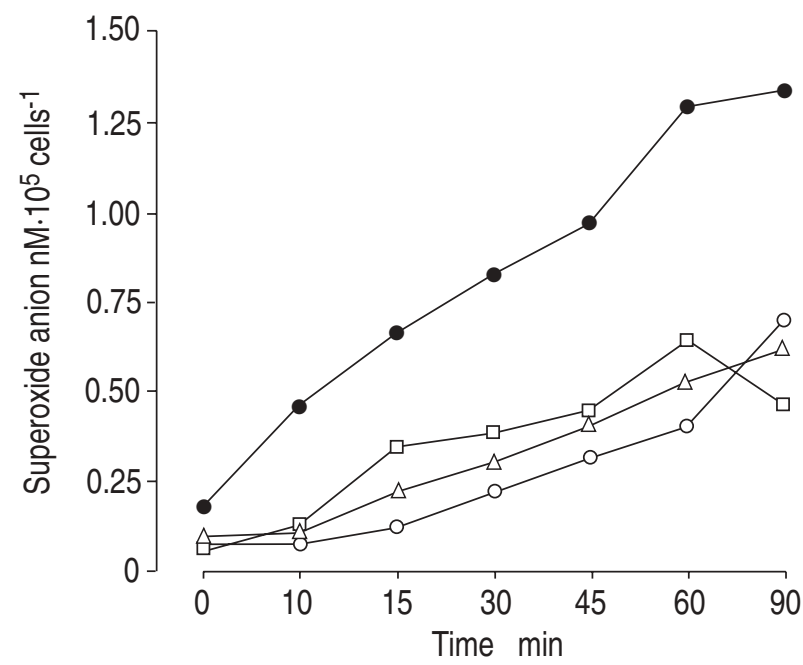

Fig. 2. - Kinetic curves for phorbol 12-myristate 13-acetate (PMA)stimulated superoxide anion production by blood monocytes. $\bullet$ : untreated; $\bigcirc$ : short-term treatment; $\square$ : steroid-dependent asthmatics; $\Delta$ : patients treated with inhaled steroids. Each symbol represents the mean of four reactions performed simultaneously.

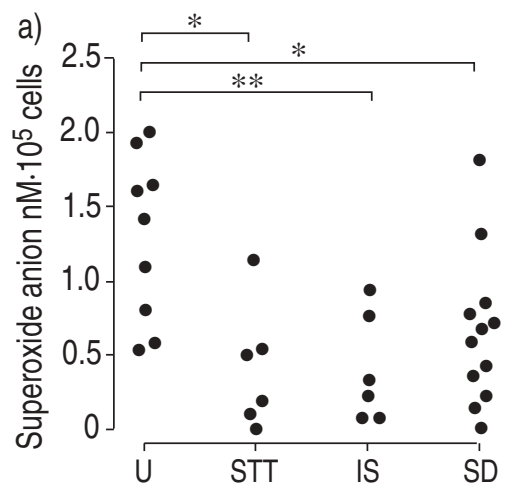

b)

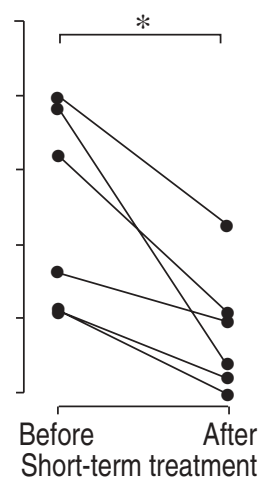

Fig. 3. - Phorbol 12-myristate 13-acetate-stimulated superoxide anion production by blood monocytes in untreated, short-term treatment, steroid-dependent asthmatics and patients treated with inhaled steroids after: a) $60 \mathrm{~min}$ stimulation; and b) before and after treatment. Each symbols represents superoxide anion production in an individual subject, and the measurements are the means of quadruplicate determinations. U: untreated; STT: short-term treatment; IS: inhaled steroids; SD: steroid dependent; *: $\mathrm{p}<0.05 ; * *: \mathrm{p}<0.01$.

tions of treated asthmatic patients as compared to untreated patients.

Concerning PMA-stimulated production, the three populations of treated asthmatic patients showed a significantly lower superoxide anion production by blood monocytes as compared to untreated asthmatic patients (fig. 3). Significant differences were observed using the Mann-Whitney U-test between untreated asthmatics and those treated with inhaled steroid $(\mathrm{p}<0.01)$ and those who were steroiddependent $(\mathrm{p}<0.02)$ and using the Wilcoxon W-test between asthmatic patients before and after treatment $(\mathrm{p}<0.05)$.

Except for steroid-dependent asthmatic patients, a significant difference in superoxide anion release by blood monocytes was observed using the Mann-Whitney U-test between untreated (uncontrolled) and short-term treated asthmatic patients and those treated with inhaled steroids (controlled) ( $<<0.02)$ (fig. 4).

The stimulatability coefficient was identical for untreated asthmatic patients and patients treated with inhaled

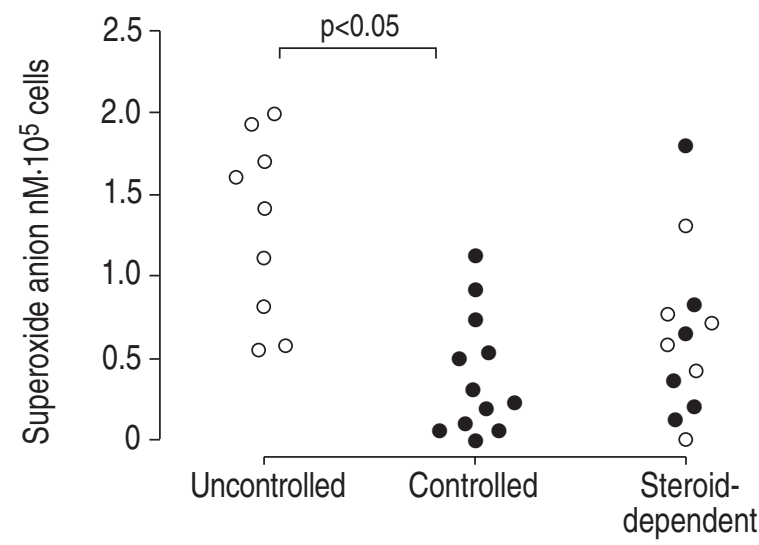

Fig. 4. - Phorbol 12-myristate 13-acetate-stimulated superoxide anion production by blood monocytes from untreated (uncontrolled), shortterm treatment and patients treated with inhaled steroids (controlled), and steroid-dependent asthmatics after $60 \mathrm{~min}$ stimulation. Each symbol represents superoxide anion production in an individual subject, and the measurements are the means of quadruplicate determinations.

steroids, with no significant difference between long-term oral and short-term $i$.v. treatment of asthmatic patients.

\section{Discussion}

This study demonstrated that long-term treatment with oral or inhaled corticosteroids and short-term treatment with high doses of corticosteroids showed reduced PMAstimulated superoxide anion production by blood monocytes from asthmatic patients as compared to untreated asthmatic patients. Spontaneous superoxide anion production was identical in untreated and treated asthmatics and was not significantly decreased in blood monocytes from inhalation treated asthmatic patients. The shapes of the kinetic curves for PMA-stimulated blood monocytes were all similar, with increasing amounts of superoxide anion production until $60 \mathrm{~min}$. A plateau was then reached for untreated asthmatic patients. Consequently, a period of 60 min was chosen to analyse individual time point levels of superoxide anion production in all groups of asthmatic patients. This analysis showed a significant decrease in superoxide anion production by blood monocytes from all treated asthmatics as compared to untreated patients. It has been demonstrated that the PMA concentration used (100 $\left.\mathrm{ng} \cdot \mathrm{mL}^{-1}\right)$ remained the optimal concentration in steroid-dependent asthmatic patients, without a shift in the dose-response curve (data not shown).

In previous work, it was observed that cells from controlled asthmatic patients produced significantly lower amounts of superoxide anion than uncontrolled ones [9]. In the present study, all untreated asthmatic patients were included on the basis of their instability, characterized by nocturnal asthma symptoms and high $\beta_{2}$-agonist usage. These patients were subjected to short-term treatment with a high dose of corticosteroids to rapidly improve their asthma symptoms, with a minimum $15 \%$ increase in their FEV1 after 10 days of therapy. After this treatment, all patients had improved symptoms, and all had a significantly decreased superoxide anion production by blood monocytes. These results could be correlated with other studies investigating the effects of corticosteroids on bronchial responsiveness [25]. 
The anti-inflammatory effects of corticosteroids on ROS inhibition in various cell types are controversial [21]. In vitro studies have suggested that the anti-inflammatory action of corticosteroids depends at least partially on an interference with the formation of reactive oxygen species by phagocytes [26].

SzEFLER et al. [27] showed that when human monocytes from normal subjects were treated with hydrocortisone in vitro, there was a significant inhibition of superoxide anion release over $32 \mathrm{~h}$. Moreover, prednisone and progesterone which are non-active corticosteroids, had no effect on this production. To our knowledge, no studies have been performed to analyse superoxide anion production from steroid-dependent asthmatic patients treated with the minimum dose of corticosteroids required in corticosteroid therapy. Our results show that superoxide anion release by blood monocytes, from steroid-dependent asthmatics or patients undergoing long-term treatment with inhaled steroids after PMA-stimulation, are quite similar to those obtained after short-term treatment with a high dose of methylprednisolone. From patients who had inhaled steroids, there was a decrease in superoxide anion production by blood cells, suggesting that they have a systemic effect or that they activate a molecule which affects monocytes progenitors in the bone marrow. However, we did not observe the same inhibitory effects of corticosteroids in patients who had been clinically well controlled with their treatment (short-term i.v. corticosteroids, or long-term inhaled corticosteroids) and in steroid-dependent asthmatic patients who were clinically different. Indeed, we did not observe a correlation between FEV1 and the release of superoxide anion by blood monocytes in steroid-dependent asthmatic patients (data not shown). These patients, who had been monitored in the department for at least $1 \mathrm{yr}$, presented with a different clinical pattern [28], and respond differently to their treatment on a longterm basis.

Interestingly, the coefficient of stimulatability for asthmatic patients with inhaled steroid treatment was the same as that obtained for untreated patients, due to the fact that the spontaneous release of superoxide anion was very low. In a previous study, we observed that in control subjects blood monocytes were not able to release any detectable amounts of superoxide anion [5]. The weak release of superoxide anion by blood monocytes from patients treated with inhaled steroids, and the fact that they were very well controlled by their treatment, suggests that superoxide anion release may reflect good control of the disease.

It is well known that anti-inflammatory effects of corticosteroids are partly mediated by the inhibition of arachidonic acid, induced by the synthesis and release of phospholipase-A2 inhibitors such as lipocortin-1 [29]. However, it is not yet clear whether corticosteroids suppress superoxide anion production by a phospholipasedependent [30] or independent [31] mechanism. The corticosteroid effects are due to a specific interaction with the glucocorticoid receptor, and the complex hormone-receptor is able to modulate the transcription of corticosteroid-responsive genes. Activation of respiratory burst involves several steps and can be induced by a variety of stimuli. The phorbol ester PMA binds and activates protein kinase $\mathrm{C}$ which is then translocated to the membrane [32]. The responses to the common three stimulators used to stimulate superoxide anion production (PMA, N-formyl- methionyl leucyl phenylalanine (fMLP) and opsonized zymosan) were affected similarly by corticosteroids [21]. This suggests that corticosteroids commonly affect components in the activation pathway, possibly protein kinase $\mathrm{C}$ or NADPH oxidase. A direct effect of dexamethasone on phagocytes has been demonstrated in vitro, leading to a dose-dependent reduction of messenger ribonucleic acid coding for the NADPH oxidase component [33]. Furthermore, corticosteroids may influence oxygen-free radicals by scavenging reactive molecules directly as superoxide dismutase, catalase or gluthation peroxidase. Corticosteroids, in addition to the suppression of reactive oxygen species production, may thus also raise intracellular levels of their scavengers [34].

In conclusion, it is interesting to note that corticosteroids administered in three different ways inhibits superoxide anion release by blood monocytes in asthmatic patients. Inhaled steroids are able to inhibit superoxide anion release by blood monocytes, suggesting an action of these inhaled steroids outside of the lungs.

\section{References}

1. Poston RN, Chanez P, Lacoste JY, Litchfield T, Lee TH, Bousquet J. Immunohistochemical characterization of the cellular infiltration in asthmatic bronchi. Am Rev Respir Dis 1992; 145: 918-921.

2. Wallaert B, Lassalle P, Fortin F, et al. Superoxide anion generation by alveolar inflammatory cells in simple pneumoconiosis and in progressive massive fibrosis of nonsmoking coal workers. Am Rev Respir Dis 1990; 141: 129-133.

3. Nordman S, Nyberg P, Linko L. Increased PMA-induced chemiluminescence from whole blood of patients with bronchial hyperreactivity. Eur Respir J 1994; 7: 14251430.

4. Jarjour NN, Calhoun WJ. Enhanced production of oxygen radicals in asthma. J Lab Clin Med 1994; 123: 131136.

5. Vachier I, Damon M, Le-Doucen C, et al. Increased oxygen species generation in blood monocytes of asthmatic patients. Am Rev Respir Dis 1992; 146: 1161-1166.

6. Cluzel M, Damon M, Chanez P, et al. Enhanced alveolar cell luminol-dependent chemiluminescence in asthma. $J$ Allergy Clin Immunol 1987; 80: 195-201.

7. Chanez P, Dent G, Yukawa T, Barnes PJ, Chung KF. Generation of oxygen free radicals from blood eosinophils from asthma patients after stimulation with PAF or phorbol ester [see comments]. Eur Respir J 1990; 3: 1002 1007.

8. Calhoun WJ, Reed HE, Moest DR, Stevens CA. Enhanced superoxide production by alveolar macrophages and air-space cells, airway inflammation, and alveolar macrophage density changes after segmental antigen bronchoprovocation in allergic subjects. Am Rev Respir Dis 1992; 145: 317-325.

9. Vachier I, Chanez P, Le-Doucen C, Damon M, Descomps B, Godard P. Enhancement of reactive oxygen species formation in stable and unstable asthmatic patients. Eur Respir J 1994; 7: 1585-1592.

10. Meltzer S, Goldberg B, Lad P, Easton J. Superoxide generation and its modulation by adenosine in the neutrophils of subjects with asthma. J Allergy Clin Immunol 1989; 83: 960-966.

11. Neijfens HJ, Raatgeep RE, Degenhart HJ, Duverman EJ, 
Kerrebijn KF. Altered leucocyte response in relation to the basic abnormality in children with asthma and bronchial hyperresponsiveness. Am Rev Respir Dis 1984; 130: 744-747.

12. Barnes PJ. Anti-inflammatory therapy for asthma. Annu Rev Med 1993; 44: 229-242.

13. Lew W, Oppenheim IJ, Matsushima K. Analysis of suppression of IL-1 alpha and IL-1 beta production in human peripheral blood mononuclear adherent cells by glucocorticoid hormone. J Immunol 1988; 140: 1895-1902.

14. Cronstein BN, Kimmel SC, Levin RI, Martiniuk F, Weissmann G. A mechanism for the anti-inflammatory effects of corticosterone: the glucocorticoid receptor regulates leucocyte adhesion to endothelial cells and expression of endothelial-leucocyte adhesion molecule 1 and intercellular adhesion molecule 1. Proc Natl Acad Sci USA 1992; 89: 9991-9995.

15. Umeki S, Soejima R. Hydrocortisone inhibits the respiratory burst oxidase from human neutrophils in whole-cell and cell-free systems. Biochim Biophys Acta 1990; 1052: 211-215.

16. Schaffner A, Schaffner T. Glucocorticoid-induced impairment of macrophage antimicrobial activity: mechanism and dependence of state of activation. Rev Infect Dis 1987; 9 (Suppl. 5): S620.

17. Haar D, Nielsen H. The effects of betamethasone, methylprednisolone and hydrocortisone on lucigenin- and luminol-amplified chemiluminescence generated in human monocytes. Scand J Rheumat 1988; 17: 487-490.

18. Fleming SD, Edelman LS, Chapes SK. Effects of corticosterone and microgravity on inflammatory cell production of superoxide. J Leukocyte Biol 1991; 50: 69-76.

19. Bergstrand $\mathrm{H}$, Bjornson A, Bkaschke E, et al. Effect of an inhaled corticosteroid, budesonide, on alveolar macrophage function in smokers. Thorax 1990; 45: 362-368.

20. Duddridge M, Ward C, Hendrick DJ, Walters EH. Changes in bronchoalveolar lavage inflammatory cells in asthmatic patients treated with high dose inhaled beclomethasone dipropionate. Eur Respir J 1993; 6(4): 489497.

21. Roshol H, Skrede KK, Aero CE, Wiik P. Dexamethasone and methylprednisolone affect rat peritoneal phagocyte chemiluminescence after administration in vivo. Eur $J$ Pharmacol 1995; 286: 9-17.

22. Lin X, Tan WC, Candlish JK. Hydrocortisone administration and superoxide production of neutrophils from asthmatic patients. Med Sci Res 1993; 21: 393-394.

23. This official statement of the American Thoracic Society was adopted by the ATS Board of Directors. Standards for the diagnosis and care of patients with chronic obstructive pulmonary disease (COPD) and asthma. November 1986. Am Rev Respir Dis 1987; 136: 225-244.

24. WHO/NHLBI workshop report. Global strategy for asthma management and prevention. National Institutes of Health, National Heart, Lung and Blood Institute, 1995; Publication Number 95-3659.

25. Jenkins CR, Woolcock AJ. Effect of prednisone and beclomethasone dipropionate on airway responsiveness in asthma: a comparative study. Thorax 1988; 43(5): 378-384.

26. Whitehouse MW, Cleland LG. Reactive oxygen species and drug therapy for inflammatory disease. Agents Actions 1985; 17: 173-177.

27. Szefler SJ, Norton CE, Ball B, Gross JM, Aida Y, Pabst MJ. IFN-gamma and LPS overcome glucocorticoid inhibition of priming for superoxide release in human monocytes. Evidence that secretion of IL-1 and tumor necrosis factor-alpha is not essential for monocyte priming. $J$ Immunol 1989; 142: 3985-3992.

28. Paradis L, Des Roches A, Vignola AM, et al. Heterogeneity of glucocorticoid (GCs) dependent asthmatics. Am J Respir Crit Care Med 1995; 151(4): A675.

29. Flower RJ, Rothwell NJ. Lipocortin-1: cellular mechanisms and clinical relevance. Trends Pharmacol Sci 1994; 15: 71-76.

30. Bromberg Y, Pick E. Unsaturated fatty acids as second messengers of superoxide generation by macrophages. Cell Immunol 1983; 79: 240-252.

31. Schultz RM, Nanda SKW, Altom MG. Effect of various inhibitors of arachidonic acid oxygenation on macrophage superoxide release and tumoricidal activity. J Immunol 1995; 135: 2040-2044.

32. Lehrer RI, Cohen L. Receptor-mediated regulation of superoxide production in human neutrophils stimulated by phorbol myristate acetate. J Clin Invest 1981; 68: 1314-1320.

33. Amezaga MA, Bazzoni F, Sorio C, Rossi F, Cassatella MA. Evidence for the involvement of distinct signal transduction pathways in the regulation of constitutive and interferon-G-dependent gene expression of NADPHoxidase components (gp91, phox, p47-phox, and p22phox) and high affinity receptor for IgG (FcgR-I) in human polymorphonuclear leukocytes. Blood 1992; 79: 735-744.

34. Youssef AA, Baron DN. Leucocyte superoxide dismutase in rheumatoid arthritis. Ann Rhem Dis 1983; 42(5): 558562. 